for drainage, as hemostasis had been well effected by ligature and hot water irrigation. The hand was dressed in an extended position upon an anterior splint. The dressing was removed upon the fourth day and the wound presented a favorable appearance, except for a small suppurating focus around one stitch. This suture was removed and after thorough disinfection with a solution of bromin and insertion of a small iodoform gauze tampon, the dressing was re-applied. The wound was irrigated the subsequent three days and I succeeded in aborting a diffuse suppuration, the wound healing intact. The sutures were removed upon the ninth day. On the twelfth day the patient could extend the three fingers upon the splint with slight effort. He speedily recovered the use of his fingers and at the end of a month was discharged from the hospital with function entirely restored. Patient could lift a glass of water to his mouth and even write with perfect ease.

Remarks:-Although I did not have the opportunity of making an examination in vivo of the regenerative process which took place, I am satisfied that there was new tendon tissue produced to the extent of over four inches. When the patient made extension, by placing a finger at the muscle extremity, the impulse was perceptible along the whole route of the tendons, showing that there was direct communication. The new fibrous cords when in action would bulge and become prominent to such an extent as to become visible through the skin. These tests were convincing proofs. This case is also of pathologic interest by reason of the sutures uniting muscle with tendon. Muscle cells are endowed with very limited intrinsic vegetative capacity, and do not extend further than an inch from the ruptured end; so that in this case proliferation from the distal extremity was the prime factor in effecting reposition, as connective tissue is the most prolific of all tissues. I wish also to dwell on the importance of making as many sutures as possible in order to construct a canal, thereby securing a line of least resistance for the embryonal cells. If I had secured each tendon with but one suture, I am certain that there would have been very probably an obliteration of the canal by neighboring tissues, and consequent failure of the operation. An interval of four inches is an unusual occurrence. It is seldom that tendons become separated for more than one or two inches at the most; and when united by this method, the operation will yield encouraging results.

\section{RAPID METHOD OF DEMONSTRATING TUBERCLE BACILLI IN SPUTA.}

\section{BY JOHN D. KALES, M.D.}

DEMONSTRATOR OF BACTERIOLOGY IN THE MEDICAL DEPARTMENT OF NORTHWESTERN UNIYERSITY.

Those who have been engaged in the demonstration of bacteria, anci the use of the various methods of staining them, have doubtless been prompted to simplify the technique as much as possible. They can thereby attain a much greater percentage of successful preparations when the work is being done by a large class in the laboratory.

A method that is carried out with ease and success by many students working together in a laboratory, must of necessity possess certain advantages that would favor its adoption by every practitioner who realizes the diagnostic value of detecting tubercle bacilli, and has the necessary optical apparatus.
The writer has for several years stained sputa for tubercle bacilli by a rapid method, one quite generally adopted at present. It consisted in using the coverglass as if it were a dish, and while holding it with a pair of forceps, the various staining fluids were boiled upon its surface, the cover-glass being held in the smokeless flame of an alcohol lamp or Bunson burner. The usual carbol-fuchsin solution was used, and decolorization accomplished by any of the wellknown means, i.e.; Orth's solution ${ }^{1}$ or nitric acid and water 1-4. If Orth's solution is used, decolorization is slower and seems to yield preparations which have little tendency to fade.

Recently the following method has been found still more convenient. However, in all methods where the flame is applied directly to a cover-glass for the purpose of heating or boiling a staining fluid, there is great need of using only the best quality of glass. This should be very thin, white and well annealed. The whiteness may be determined by looking at the edges of a package of glasses; these should show no greenish tinge. Their brittleness may be estimated by bending one between the fingers; it should stand considerable flexion before breaking. In buying coverglasses it is necessary to observe these conditions, for a large number that are sold are not fit to use in rapid staining methods where the flame is directly applied.

It is also very important that the forceps used for holding the cover-glass be of the right kind. The best are the curved fixation forceps made for oculists. Their blades are so set that fluids are not drawn between them by capillary attraction. They grasp the cover-glass firmly and may be locked. The teeth are of no use and can be smoothed off with a file.

One should be provided with a saturated alcoholic solution of fuchsin, which is made by adding dry fuchsin to a quantity of alcohol until no more will be dissolved; the excess will settle to the bottom of the fluid and should be allowed to remain there. A pipette bottle is most convenient for this solution, which keeps indefinitely. A 5 per cent. solution of carbolic acid in distilled water should be at hand and is best kept in a pipette bottle also. For decolorizing, a 25 per cent. solution of nitric acid is placed in a wine glass.

Suppose we use a thin, clean and perfectly dry cover-glass of good quality, three-fourths of an inch square, the film of sputum being fixed upon it in the usual manner; then it will be found that twelve drops of the 5 per cent. carbolic acid solution will cover its surface. This is dropped on while the glass is held in the forceps, then with care the fuchsin solution is added, drop by drop. The first two drops make a blocd red precipitate in the carbolic acid solution, the third tends to clear this slightly, while the fourth should cause the precipitate to disappear entirely. Thus when twelve drops of the 5 per cent. carbolic acid are used, four drops of fuchsin will be found necessary. Other sizes of cover-glasses will require a different quantity of the stain, though the proportion between the two fluids will be practically maintained as 4-12 or 1-3. Whatever size glass is used it must be completely covered with the carbolic acid solution, and it is well to add a few extra drops. This will prevent evaporation leaving any portion of the glass dry. We may ignore the above proportions

1 Strong hydrochloric acid, 1 part; alcohol, 30 parts; water, 70 parts. 
and always drop the fuchsin until the precipitate that first forms, clears.

Holding the cover-glass in suitable forceps, the staining fluid is boiled upon its surface, but never long enough to dry on the glass. Wash the glass in a stream of running water, still holding it with the forceps, and then wave it back and forth in the decolorizing solution until all color is lost; this may take twenty or thirty seconds. Wash again in running water, dry with the help of blotting paper and heat. If desired, counter stain with cold saturated aqueous solution of methyl blue; if not, invert the dry glass on a drop of glycerin, balsam or water for examination.

The entire process of fixing, staining, decolorizing, etc., may take from two to four minutes, and during this time the glass has been held continuously in the forceps.

There may be conditions when the application of a cold stain for tubercle bacilli-the cover-glass being left in the fluid for twenty-four hours-would be advantageous. The writer has seldom found such conditions necessary. But the above rapid process, which obviates the necessity for keeping on hand staining solutions liable to deteriorate, or obliges one to make up a stock solution whenever there is a suspicion that the one on hand has grown too old, has its advantages.

The overcoming of these difficulties may induce the general practitioner to avail himself more frequently of that method of detecting tuberculosis which has now grown to have a very considerable importance.

\section{AN ABDOMINO-INGUINAL SUPPORTER. FOR USE AFTER HERNIOTOMY.}

\section{BY JOHN B. HAMILTON, M.D., LLD.}

PROFESSOR PRINCIPLES OF SURGERY AND CLINICAL SURGERY RUSH MEDICAL COLLEGE; PROFESSOR SURGERY CHICAGO POLICLINIC.

"The patient should not be allowed to get up until three weeks have elapsed after the operation and then only if the wound is sound. The question of a supporting bandage or a truss will then have to be considered."-Treves, "Manual of Operative Surgery," 1892, Vol. 2, page 530.

"A spring truss should not be worn after the operation as its pressure tends to cause premature absorption of the lymph, but the abdominal wall requires a certain amount of support for some months. In infants this may be managed with a skein of Berlin wool as already described; but for children and adults the form of appliance known as Harrison's truss answers best. It consists of a well fitting pelvic band and two short thigh preces made of linen; like a pair of very short drawers fitting tightly and fastened with lacers; a perineal band prevents its ascending, and may in addition be furnished with a scrotal bag and with braces passing over the shoulders."-Moullin, "Surgery," $2 \mathrm{~d}$ Ed. by Hamilton, 1894 , p. 904.

"In all cases during more than six months, those who are subjected to the radical cure should be watched with care and on the first menace of the reappearance of the hernia a bandage must be prescribed, so as not to lose all the benefits of the operation.-Berger, Traité de Chirurgie, Duplay et Reclus, Tome vi.

My experience, now quite extensive, in operations for the radical cure of hernia, has convinced me of the general correctness of the view, that some kind of support is always indicated after herniotomy. The direction given in some of the recent writings on the subject, to cause the patient to wear a light spring truss for some weeks, is only less mischievous than the practice of discharging such patients without any support. In casting about for some apparatus that would approach the shape of the human hand in supporting the new cicatricial tissue closing the inguinal ring, I thought a thin piece of flat sole leather slightly hollowed like the palm of the hand, covered with chamois skin, making uniform pressure on the abdomen, would fulfil the indications. To retain the broad pad in place I caused it to be sewed to a supporter made of silk elastic, with a thigh piece."

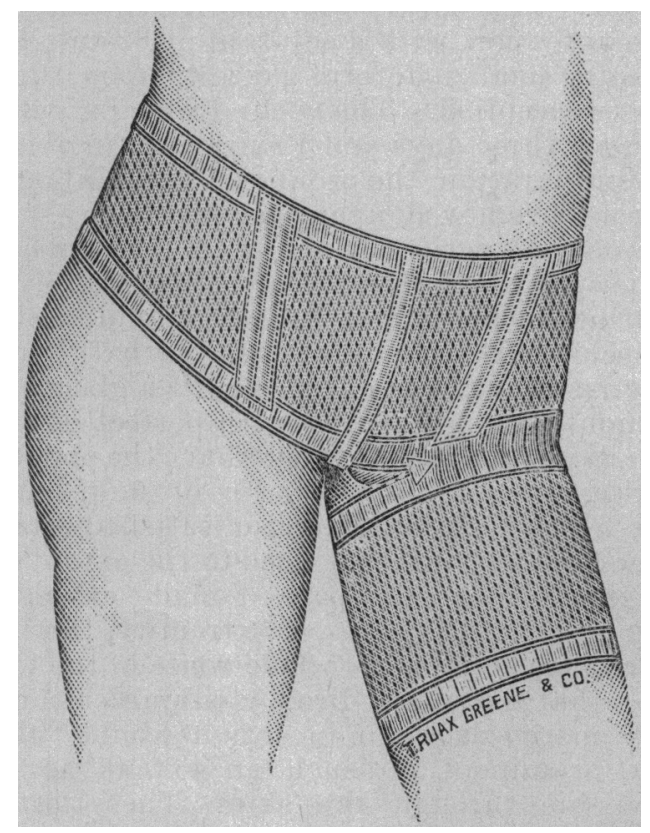

The cut shows the supporter as made for me by Truax, Greene \& Co.

I have tried it on three cases, and with satisfaction to the patient in all.

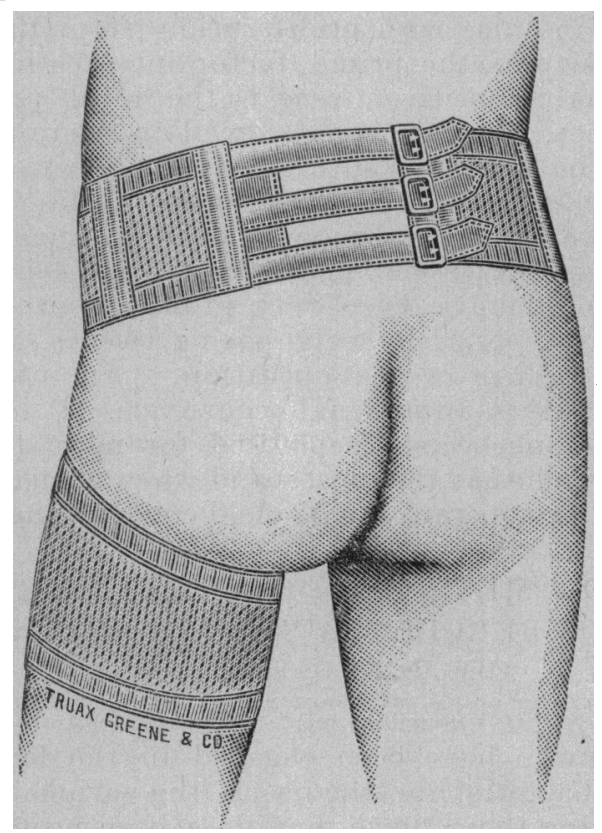

With this bandage the pendulous abdomen may be well sustained, and the thigh piece keeps the pad in position. The bandage should be woven in a single piece, and the pad carefully fitted to the patient before permanent fastening. Care should be taken that the thigh piece is not too tight. The straps should be snugly buckled at the back. Patients wearing these supporters have been instructed to remove them at night.

Buena Park, Chicago, April 20, 1894. 